\title{
Association of Low-Dose Glucocorticoid Use And Infection Occurrence in Systemic Lupus Erythematosus Patients: A Prospective Cohort Study
}

\section{Kazuya Abe}

Yokohama Rosai Hospital: Yokohama Rosai Byoin

Yuichi Ishikawa ( $\square$ ishikawa.u1@gmail.com)

University of Occupational and Environmental Health, Japan https://orcid.org/0000-0001-7406-2309

Yasuhiko Kita

Yokohama Rosai Hospital

Nobuyuki Yajima

Showa University

Eisuke Inoue

Showa University

\section{Ken-ei Sada}

Kochi Medical School, Kochi University

\section{Yoshia Miyawaki}

Okayama University

\section{Ryusuke Yoshimi}

Yokohama City University

\section{Yasuhiro Shimojima}

Shinshu University

\section{Shigeru Ohno}

Yokohama City University Medical Center

Hiroshi Kajiyama

Saitama Medical University: Saitama Ika Daigaku

Kunihiro Ichinose

Nagasaki University: Nagasaki Daigaku

\section{Shuzo Sato}

Fukushima Medical University Aizu Medical Center: Fukushima Kenritsu Ika Daigaku Aizu Iryo Center Michio Fujiwara

Yokohama Rosai Hospital 
Research article

Keywords: glucocorticoids, infection, prospective cohort study, systemic lupus erythematosus

Posted Date: October 28th, 2021

DOI: https://doi.org/10.21203/rs.3.rs-1009539/v1

License: (c) (i) This work is licensed under a Creative Commons Attribution 4.0 International License. Read Full License 


\section{Abstract}

Background: Infection is a major cause of mortality in patients with systemic lupus erythematosus (SLE). Therefore, minimizing the risk of infection is an important clinical goal to improve the long-term prognosis of SLE patients. Treatment with $\geq 7.5 \mathrm{mg}$ prednisolone (PSL) or equivalent has been reported to increase the risk of infections. However, it remains unclear whether $<7.5 \mathrm{mg}$ PSL or equivalent dose affects the risk of infection in SLE patients. This study evaluated the association between the occurrence of infection in patients with SLE and low-dose glucocorticoids (GC) usage, especially $<7.5 \mathrm{mg}$ PSL or equivalent, to explore the GC dose that could reduce infection occurrence.

Methods: This prospective cohort study included patients from the Japanese multicenter registry of patients with SLE (defined as $\geq 4$ American College of Rheumatology 1997 revised criteria) over 20 years of age. The PSL dose was categorized as PSL $0-2.5,2.6-5.0,5.1-7.5$, and $7.6-15.0 \mathrm{mg}$. The primary outcome was infection requiring hospitalization. We conducted a multivariable analysis using timedependent Cox regression analysis to assess the hazard ratio of infection occurrence compared with a dose of $0-2.5 \mathrm{mg}$ PSL or equivalent in the other three PSL dose groups. Based on previous reports and clinical importance, the covariates selected were age, sex, and concurrent use of immunosuppressants with GC. In addition, two sensitivity analyses were conducted.

Results: The mean age of the 509 SLE patients was 46.7 years; $89.0 \%$ were female, and $77.2 \%$ used multiple immunosuppressants concomitantly. During the 1-year observation period, infection requiring hospitalization occurred in 52 patients. The incidence of infection with a PSL dose of 5.0-7.5 mg was significantly higher than that in the PSL $0-2.5 \mathrm{mg}$ group (adjusted hazard ratio: $6.80,95 \%$ confidence interval: $2.17-21.27)$. The results of the two sensitivity analyses were similar.

Conclusions: Our results suggested that the use of 5.0-7.5 mg PSL or equivalent could pose an infection risk in SLE patients. This finding indicated that PSL dose should be reduced to as low as possible in SLE patients to avoid infection.

\section{Background}

Infection is a major cause of mortality in patients with systemic lupus erythematosus (SLE).[1, 2] Therefore, minimizing the risk of infection is an important clinical goal to improve the prognosis of SLE patients. Previous studies have suggested that the use of prednisolone (PSL) at a dose $\geq 7.5 \mathrm{mg}$, high SLE disease activity, intravenous administration of cyclophosphamide, a history of lupus nephritis, decreased white blood cell count, anti-dsDNA IgG levels $>20 \mathrm{IU} / \mathrm{ml}$, and decreased complement levels were risk factors for infection in SLE patients.[3-6]

Regarding the risk of infection with glucocorticoids (GCs), previous exploratory studies have suggested that daily PSL at a dose higher than $7.5 \mathrm{mg}$ posed a severe infection risk in SLE patients. [7-9] On the contrary, PSL dose $\leq 5 \mathrm{mg}$ has been reported to increase infection risk in rheumatoid arthritis patients.[10] In addition, a cohort study assessing the risk of hospitalization in patients with rheumatic disease and 
COVID-19 found that >5 mg PSL was associated with higher odds for hospitalization.[11] These studies showed that even doses of PSL $<7.5 \mathrm{mg}$ could be risk factors for infection and hospitalization in patients with rheumatic disease. Thus, we hypothesized that a PSL dose $<7.5 \mathrm{mg}$ could pose an infection risk in SLE patients, as with other rheumatic diseases.

However, no study to date has assessed whether PSL $<7.5 \mathrm{mg}$ daily or equivalent GC dose increases the infection risk in SLE patients. In addition, the lupus low disease activity state (LLDAS) has recently been described as a clinically relevant treatment target in SLE patients, and current PSL or equivalent dose $\leq 7.5 \mathrm{mg}$ is one of the LLDAS criteria.[12] LLDAS attainment has been reported to decrease organ damage induced by SLE.[13] However, it is unknown whether achieving this target PSL dosage could reduce infection in SLE patients.

If a PSL dose $<7.5 \mathrm{mg}$ poses an infection risk in SLE patients, it is necessary to achieve maintenance therapy with a PSL dose lower than the currently recommended dose. This study investigated the relationship between low-dose GC ( $\leq 7.5 \mathrm{mg}$ PSL or equivalent) and infection occurrence in SLE patients, using a database of the multicenter registry of SLE patients in Japan.

\section{Methods}

\section{Aim}

This study aimed to investigate the relationship between low-dose GC ( $\leq 7.5 \mathrm{mg}$ PSL or equivalent) and infection occurrence in SLE patients, using a database of the multicenter registry of SLE patients in Japan.

\section{Study design}

This study was a prospective cohort study using data from the Lupus Registry of Nationwide Institutions (LUNA), a multicenter database of patients with SLE in Japan.

\section{Setting}

The participating institutions included the rheumatology departments of eight university hospitals and one general hospital. Because this study included data from multiple institutions from various regions of Japan, we were able to minimize selection bias and achieve geographical representativeness.

We collected data about GC use during the study period, and data regarding other patient characteristics were collected once a year at each hospital. Patient data were collected until the patient's death, transfer out of the hospital, or patient opt-out from the registry.

\section{Participants}

Patients were recruited from February 2016 to September 2019. We included SLE patients over 20 years who fulfilled the American College of Rheumatology criteria for SLE classification,[14] including patients 
with SLE complicated with other connective tissue diseases (such as Sjogren's syndrome). The included patients were followed up for more than 1 year. We excluded patients who received daily PSL >15 mg or equivalent during the study period because this dose is already known to be an infection risk, and our focus was on the relationship between low-dose PSL and infection. Patients with missing data regarding $\mathrm{GC}$, infection, or follow-up were also excluded.

\section{Variables}

We recorded the following from the database: age, sex, comorbid diabetes mellitus (defined by a serum $\mathrm{HbA1c}>6.5 \%$ ), biopsy-proven lupus nephritis (Class $\nabla, \nabla$, and $\nabla$ according to the classification of the International Society of Nephrology and the Renal Pathology Society 2003 [15]), the SLE Disease Activity Index 2000,[16] blood serum data (C3, C4, CH50, anti-ds-DNA IgG antibody, and IgG), white blood cell count, concurrent use of multiple immunosuppressants use with GC (tacrolimus, cyclosporine, mycophenolate mofetil, azathioprine, and mizoribine), past use of intravenous immunosuppressant (methylprednisolone pulse therapy, intravenous cyclophosphamide, and rituximab), and hydroxychloroquine (HCQ) use. We recorded variables at the time of registration, and time-varying variables were also collected once a year when the patients were followed up in the registry.

\section{Primary exposure}

The primary exposure was systemic GC administration. The GC dose was averaged to the daily dose and converted to the PSL dose. The PSL dose was categorized as PSL $0-2.5,2.6-5.0,5.1-7.5$, and 7.6-15.0 $\mathrm{mg}$, as previously described.[17] Moreover, we considered this categorization to be clinically relevant as it is unknown whether PSL doses $<7.5 \mathrm{mg}$ could pose an infection risk, and the LLDAS aims to use a PSL dose $<7.5 \mathrm{mg}$.

\section{Outcome measure}

The primary outcome was infection requiring hospitalization. This outcome was selected as it is clinically relevant for patients; further, infections requiring hospitalization are usually severe and may require changes in the therapy plan. Data on hospitalizations for infection were identified from the records of the hospitals participating in this study. Hospital records were also reviewed to verify whether patients were admitted to any other hospitals not participating in LUNA. Infections were classified as respiratory infection, urinary tract infection, abdominal infection, soft tissue and bone infection, neurological infection, and others. In patients with multiple infections, each infection was considered and evaluated.

\section{Statistical analysis}

Age, SLE Disease Activity Index, blood serum data, and white blood cell count were used as continuous variables. Sex, comorbid diabetes mellitus, lupus nephritis, PSL dose group, concurrent use of multiple immunosuppressants, past use of intravenous immunosuppressive therapy, immunosuppressive therapy, HCQ use, and infection occurrence were the categorical variables analyzed. Summary statistics are presented as mean values with standard deviations and as numbers with proportions. 
We conducted a multivariable analysis using time-dependent Cox regression analysis to assess the hazard ratio of infection occurrence with PSL dose, referenced to PSL or equivalent dose of $0-2.5 \mathrm{mg}$. The covariates selected were age, sex, and concurrent immunosuppressant use, because these variables are clinically important for infection occurrence in SLE patients, as previously reported.[18-20] The covariate data were collected at registration and every other year.

We conducted two sensitivity analyses. First, we conducted a time-dependent Cox regression analysis among the patients who did not receive HCQ, because HCQ has a protective effect against infection in SLE.[21] Second, we conducted a Cox regression analysis for infection risk with total PSL dose in 1 year in each group among the patients whose PSL dose did not change over the course of 1 year. This was because the PSL dose was changed every hospital visit and therefore we were unable to accurately determine the effect of GC after every dose change. We conducted the same analysis as the primary analysis in the sensitivity analyses.

We assumed that data were missing at random, and thus missing data were handled by using multiple imputations, a method for handling missing data in epidemiological and clinical research, for multivariate analysis. Statistical significance was defined as a two-sided $p$-value $<0.05$. All statistical analyses were conducted using STATA 15.1 (Stata Corp LP, College Station, TX)

\section{Patient and public involvement}

Patients and the public were not involved in the design, conduct, and reporting of this research.

\section{Results}

\section{Study population}

A total of 573 SLE patients were extracted from the database. Sixty-four patients were excluded because they were administered $>15 \mathrm{mg}$ PSL or equivalent dose within 1 year $(n=50)$ or had missing data on days of PSL administration $(n=14)$. No patients had missing data about the PSL dose and infection occurrence. Consequently, 509 patients were included in the analysis of the relationship between PSL dose and infection occurrence (Figure 1).

\section{Baseline characteristics}

Table 1 shows the baseline characteristics of included patients. On average, participants were middleaged, and most of them were females. These results were similar to those of a previous epidemiological study.[22] The total follow-up time was 946.2 patient-years, and the mean follow-up duration in each patient was 675.3 days. The follow-up times in each group were 939.9 patient-years in the PSL $0-2.5 \mathrm{mg}$, 939.5 patient-years in the PSL 2.6-5.0 mg, 931.6 patient-years in the PSL $5.1-7.5 \mathrm{mg}$, and 929.3 patientyears in the PSL 7.6-15.0 mg groups. 
Table 1

Patient characteristics

\begin{tabular}{|c|c|c|}
\hline & Patients $(n=509)$ & Missing data \\
\hline Age (years), mean $\pm S D$ & $46.7 \pm 14.8$ & 0 \\
\hline Sex (female), n (\%) & $453(89.0 \%)$ & 0 \\
\hline Follow-up days, mean \pm SD & $675.3 \pm 343.3$ & 0 \\
\hline mPSL pulse therapy, $\mathrm{n}(\%)$ & $197(38.7 \%)$ & 0 \\
\hline Immunosuppressant, n (\%) & $393(77.2 \%)$ & 0 \\
\hline Cyclophosphamide, n (\%) & $130(25.5 \%)$ & 0 \\
\hline Tacrolimus, n (\%) & $164(32.2 \%)$ & 0 \\
\hline Cyclosporin, n (\%) & $30(5.9 \%)$ & 0 \\
\hline Mycophenolate mofetil, n (\%) & $61(12.0 \%)$ & 0 \\
\hline Azathioprine, n (\%) & $76(14.9 \%)$ & 0 \\
\hline Mizoribine, n (\%) & $21(4.1 \%)$ & 0 \\
\hline Methotrexate, n (\%) & $18(3.5 \%)$ & 0 \\
\hline Rituximab, n (\%) & $9(1.8 \%)$ & 0 \\
\hline Hydroxychloroquine, n (\%) & $87(17.1 \%)$ & 0 \\
\hline WBC $(/ \mu \mathrm{L})$, mean $\pm \mathrm{SD}$ & $5815.1 \pm 2108.8$ & $1(2.0 \%)$ \\
\hline $\mathrm{HbA} 1 \mathrm{c}>6.5 \%$ & $24(4.7 \%)$ & $105(20.6 \%)$ \\
\hline 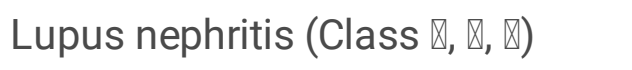 & $127(25.0 \%)$ & $21(4.1 \%)$ \\
\hline \multicolumn{3}{|l|}{ (Biopsy was performed 195/509) } \\
\hline Current smoker & $118(23.2 \%)$ & $28(5.5 \%)$ \\
\hline SLEDAI score & $5.2 \pm 4.6$ & $49(9.6 \%)$ \\
\hline C3 $(\mathrm{mg} / \mathrm{dL})$, mean $\pm \mathrm{SD}$ & $83.1 \pm 22.3$ & $23(4.5 \%)$ \\
\hline C4 $(\mathrm{mg} / \mathrm{dl})$, mean $\pm \mathrm{SD}$ & $17.0 \pm 8.8$ & $11(2.1 \%)$ \\
\hline $\mathrm{CH} 50(\mathrm{U} / \mathrm{ml})$, mean $\pm \mathrm{SD}$ & $35.6 \pm 10.9$ & $13(2.6 \%)$ \\
\hline Anti-ds DNA (IU/ml), mean \pm SD & $20.8 \pm 36.6$ & $23(4.5 \%)$ \\
\hline $\operatorname{lgG}(\mathrm{mg} / \mathrm{dl})$, mean $\pm \mathrm{SD}$ & $1411.7 \pm 478.6$ & $57(11.2 \%)$ \\
\hline
\end{tabular}

SD: standard deviation; WBC, white blood cell count; SLEDAI, SLE Disease Activity Index; Anti-ds DNA, anti-double strand DNA antibody 


\begin{tabular}{|lcc|}
\hline & Patients $(\mathbf{n}=\mathbf{5 0 9})$ & Missing data \\
\hline Pneumococcal vaccination & $57(11.1 \%)$ & $23(4.5 \%)$ \\
\hline $\begin{array}{l}\text { SD: standard deviation; WBC, white blood cell count; SLEDAl, SLE Disease Activity Index; Anti-ds DNA, } \\
\text { anti-double strand DNA antibody }\end{array}$ & \\
\hline
\end{tabular}

\section{Incidence of infection}

There were 52 infection occurrences during the study period (Table 2).

Table 2

\begin{tabular}{|c|c|c|}
\hline & Infection occurrence & Percentage \\
\hline Respiratory & 25 & $48.1 \%$ \\
\hline Urinary tract & 12 & $23.1 \%$ \\
\hline Abdominal & 10 & $19.2 \%$ \\
\hline Soft tissue & 3 & $5.8 \%$ \\
\hline Others & 2 & \\
\hline
\end{tabular}

Most infections were respiratory (48.1\%), followed by urinary tract infections $(23.1 \%)$, and abdominal infections (19.2\%).

\section{Associations between PSL dose and infection occurrence}

The adjusted hazard ratio of infection occurrence compared with the PSL 0-2.5 mg group was 2.69 (95\% $\mathrm{Cl} 0.90-7.99)$ in the PSL 2.6-5.0 mg group, $6.80(95 \% \mathrm{Cl} 2.17-21.27)$ in the PSL 5.1-7.5 $\mathrm{mg}$ group, and 7.68 (95\% Cl 2.38-24.85) in the PSL 7.6-15.0 mg group (Figure 2).

\section{Sensitivity analysis}

The results of the two sensitivity analyses are presented in Table 3 and 4. 
Table 3

Hazard ratio for infection occurrence in patients who were not administered hydroxychloroquine.

\begin{tabular}{|lll|}
\hline Glucocorticoid dose & \multicolumn{2}{l|}{ Hazard ratio for infection occurrence (95\% confidence interval) } \\
\cline { 2 - 3 } (PSL or equivalent) & Unadjusted & Adjusted \\
\hline PSL $0-2.5 \mathrm{mg}$ & Reference & Reference \\
\hline PSL 2.6-5.0 mg & $2.27(0.76-6.80)$ & $2.51(0.83-7.59)$ \\
\hline PSL 5.1-7.5 mg & $3.81(1.17-12.43)$ & $5.28(1.57-17.77)$ \\
\hline PSL 7.6-15.0 mg & $5.46(1.72-17.38)$ & $8.56(2.55-28.72)$ \\
\hline
\end{tabular}

Table 4

Hazard ratio for infection occurrence in patients with fixed PSL dose for 1 year.

\begin{tabular}{|lll|}
\hline Glucocorticoid dose & Hazard ratio for infection occurrence (95\% confidence interval) \\
\cline { 2 - 3 } (PSL or equivalent) & Unadjusted & Adjusted \\
\hline PSL 0-2.5 mg & Reference & Reference \\
\hline PSL 2.6-5.0 mg & $6.78(0.88-52.56)$ & $6.97(0.89-54.41)$ \\
\hline PSL 5.1-7.5 mg & $9.15(1.02-81.99)$ & $9.67(1.07-87.17)$ \\
\hline PSL 7.6-15.0 mg & $6.58(0.68-63.27)$ & $7.53(0.75-75.54)$ \\
\hline
\end{tabular}

First, the time-dependent Cox regression analysis among 508 patients who were not administered HCQ revealed that PSL $>5.0 \mathrm{mg}$ increased the risk of infection compared with PSL $0-2.5 \mathrm{mg}$ (Table 3). This result of time-dependent Cox regression analysis was consistent with that of the main analysis. Second, the Cox regression analysis among 329 patients whose PSL dose did not change in 1 year showed that a PSL dose of $5.1-7.5 \mathrm{mg}$ increased the risk of infection compared with a dose of $0-2.5 \mathrm{mg}$ (Table 4). However, the risk of infection was not significantly higher in patients treated with a PSL dose of 7.6-15.0 mg.

\section{Discussion}

This study investigated the association between a low GC dose and infection occurrence in patients with SLE, using a multicenter registry database in Japan. The results suggested that, compared with a PSL dose of $0-2.5 \mathrm{mg}$, administration of PSL $5.0-7.5 \mathrm{mg}$ increased the infection risk in SLE patients. Our findings indicated that reducing the PSL dose to less than PSL $5.0 \mathrm{mg}$ or equivalent may be necessary to decrease infection occurrence.

In this study, the hazard ratio of infection occurrence in SLE patients administered $5.0-7.5 \mathrm{mg}$ PSL was 6.80 when compared to those administered with 0-2.5 mg PSL. Hence, even 5.0-7.5 mg PSL dose could pose an infection risk in SLE patients. Previous exploratory research showed that a PSL dose $>7.5 \mathrm{mg}$ increased the risk of infection in SLE patients, [20] and this was consistent with our observations. 
Unlike previous reports which did not find any increased risk of infection with lower-dose PSL, our observations may be explained by two main reasons. First, this may have been due to differences in statistical analysis. Our study was analyzed using a time-dependent Cox regression analysis, whereas previous studies assessed the effect of GC on infection based on the highest GC dose administered in the past and the GC dose at the time of study registration. $[8,9]$ Because the GC dose is often changed at each hospital visit, previous studies did not assess the effect of the current GC dose on infection correctly. In contrast, our study collected data on the GC dose at each hospital visit, and we assessed the effect of GC on infection by time-dependent Cox regression analysis. Therefore, ours was a more accurate evaluation of the relationship between infection occurrence and GC dose. Second, previous studies were exploratory and retrospective, $[8,9]$ while our study was exploratory and prospective. Therefore, we were able to estimate confounding factors and had fewer missing data. Thus, our results more accurately assessed the relationship between infection and GC dose.

Several mechanisms can explain the increased risk for infection with low-dose GC. GCs have various effects on inflammatory and immunologically mediated processes, such as impeding the access of neutrophils and monocytes to inflammatory sites and also cause lymphocytopenia.[23] These effects proved the infection occurrence. However, in an in vitro experiment, only middle- to high-dose GC doses impaired granulocyte phagocytosis.[24] Nevertheless, the results of our own and another study[10] indicate that low-dose GC can predispose to infection. Thus, low-dose GC appears to have an effect on immunity; however, further studies are required to elucidate the underlying mechanism.

Our study had several strengths. First, our study was conducted using a multicenter registry. Therefore, our study results can be generalized to most patients with SLE and current clinical practice. Second, we adjusted for confounding factors affecting infection occurrence based on previous reports. Therefore, our study allowed for a precise evaluation of the correlation between GC and infection risk in patients with SLE.

This study also had several limitations. First, we could not assess each immunosuppressant. Nonetheless, it was reported that infection risk did not differ among SLE patients treated with mycophenolate mofetil, azathioprine, and cyclophosphamide.[25] Hence, the lack of a classification of immunosuppressants is unlikely to have had marked effects. Second, we could not evaluate GC accumulation. Accumulation of the GC dose has been reported to be related to infection risk.[26] Nevertheless, the recent GC dose has a greater impact on infection than the accumulated GC dose in rheumatoid arthritis patients.[27] Thus, this limitation is unlikely to have had a marked effect on our findings. Third, we could not adjust for the effect of HCQ on infection occurrence. HCQ has been reported to have a protective role against infection.[21] Thus, our analysis may underestimate the relationship between GC and infection occurrence. However, the rate of HCQ use in our registry was low because HCQ has only recently become available for use in Japan. In addition, we conducted a sensitivity analysis to assess the association of low PSL dose and infection occurrence in patients with SLE who were not administered HCQ, and this sensitivity analysis yielded a similar result to the main analysis. Fourth, pneumococcal vaccination was not assessed as a confounding factor. It has been reported that 
pneumococcal infections are frequent and severe in patients with SLE.[20, 28] Pneumococcal vaccination efficacy in SLE patients has been established.[29] However, we could not adjust for pneumococcal vaccination status because of the patient number. Nevertheless, the number of vaccinated patients was only $11.1 \%$, and thus the influence was likely to be minimal. Fifth, most of the patients in this registry were Japanese. Therefore, our study results may not be generalizable to non-Japanese patients.

\section{Conclusions}

Our time-dependent Cox regression analysis of data from the SLE registry in Japan suggested that even low-dose GC, 5.0-7.5 mg PSL or equivalent, could increase the infection risk in SLE patients. This finding indicated that PSL dose should be reduced to as low as possible in SLE patients to avoid infection.

\section{Abbreviations}

PSL = prednisolone

SLE = systemic lupus erythematosus

$\mathrm{GC}=$ glucocorticoid

LLDAS = lupus low disease activity state

LUNA = Lupus Registry of Nationwide Institutions

$\mathrm{HCQ}=$ hydroxychloroquine

\section{Declarations}

\section{Ethical approval and consent to participate}

Informed consent was obtained from all participants prior to enrollment in the study. Patient information was anonymized and de-identified prior to analysis. This study was conducted in accordance with the tenets of the Declaration of Helsinki and the Ethical Guidelines for Medical and Health Research Involving Human Subjects in Japan. The Ethics Committee of the Yokohama Rosai Hospital approved the study protocol (\#31-35).

\section{Consent for publication}

Not applicable.

\section{Availability of data and materials}

The datasets used and analyzed during the current study are available from the corresponding author on reasonable request. 


\section{Competing interests}

RY received speaker's fees from Sanofi K.K., GlaxoSmithKline K.K., Novartis Pharmaceuticals, AYUMI Pharmaceutical Corporation, and Astellas Pharma Inc. KS received speaker's fee from Glaxo Smith Kline K.K. El received speaker's fees from Pfizer Japan Inc. and Bristol-Myers Squibb Company.

\section{Funding}

This research was not supported by any specific grants from any funding agencies in the public, commercial, or not-for-profit sectors.

\section{Author's contributions}

KA contributed to the conceptualization of the study, analysis of dataset, visualization, and manuscript draft preparation. YI contributed to the conceptualization of the study, supervision and proof reading of the manuscript. NY contributed to the conceptualization of the study, analysis of dataset, supervision and proof reading of the manuscript. El contributed to the analysis of dataset and proof reading of the manuscript. YK, KS, YM, RY, YS, SO, HK, KI, SS, and MF contributed to the manuscript review and editing.

\section{Acknowledgment}

We would like to acknowledge our colleagues for collecting the data for the registry.

\section{References}

1. Kang KY, Kwok SK, Ju JH, Park KS, Cho CS, Kim HY, et al. The causes of death in Korean patients with systemic lupus erythematosus over 11 years. Lupus. 2011;20:989-97.

2. Feng X, Pan W, Liu L, Wu M, Ding F, Hu H, et al. Prognosis for hospitalized patients with systemic lupus erythematosus in China: 5-year update of the Jiangsu cohort. PLoS ONE. 2016;11:e0168619.

3. Danza A, Ruiz-Irastorza G. Infection risk in systemic lupus erythematosus patients: susceptibility factors and preventive strategies. Lupus. 2013;22:1286-94.

4. Petri M, Genovese M. Incidence of and risk factors for hospitalizations in systemic lupus erythematosus: a prospective study of the Hopkins lupus Cohort. J Rheumatol. 1992;19:1559-65.

5. Duffy KN, Duffy CM, Gladman DD. Infection and disease activity in systemic lupus erythematosus: a review of hospitalized patients. J Rheumatol. 1991;18:1180-4.

6. Pryor BD, Bologna SG, Kahl LE. Risk factors for serious infection during treatment with cyclophosphamide and high-dose corticosteroids for systemic lupus erythematosus. Arthritis Rheum. 1996;39:1475-82.

7. Hiraki LT, Feldman $\mathrm{CH}$, Marty FM, Winkelmayer WC, Guan H, Costenbader KH. Serious infection rates among children with systemic lupus erythematosus enrolled in Medicaid. Arthritis Care Res (Hoboken). 2017;69:1620-26. 
8. Ruiz-Irastorza G, Olivares N, Ruiz-Arruza I, Martinez-Berriotxoa A, Egurbide MV, Aguirre C. Predictors of major infections in systemic lupus erythematosus. Arthritis Res Ther. 2009;11:R109.

9. Pimentel-Quiroz VR, Ugarte-Gil MF, Harvey GB, Wojdyla D, Pons-Estel GJ, Quintana R, et al. Factors predictive of serious infections over time in systemic lupus erythematosus patients: data from a multi-ethnic, multi-national, Latin American lupus cohort. Lupus. 2019;28:1101-10.

10. George MD, Baker JF, Winthrop K, Hsu JY, Wu Q, Chen L, et al. Risk for serious infection with low-dose glucocorticoids in patients with rheumatoid arthritis: A cohort study. Ann Intern Med. 2020;173:87078.

11. Hasseli R, Mueller-Ladner U, Hoyer BF, Krause A, Lorenz HM, Pfeil A, et al. Older age, comorbidity, glucocorticoid use and disease activity are risk factors for COVID-19 hospitalisation in patients with inflammatory rheumatic and musculoskeletal diseases. RMD Open. 2021;7:e001464.

12. Franklyn K, Lau CS, Navarra SV, Louthrenoo W, Lateef A, Hamijoyo L, et al. Definition and initial validation of a Lupus Low Disease Activity State (LLDAS). Ann Rheum Dis. 2016;75:1615-21.

13. Petri M, Magder LS. Comparison of remission and lupus low disease activity state in damage prevention in a United States systemic lupus erythematosus cohort. Arthritis Rheumatol. 2018;70:1790-95.

14. Hochberg MC. Updating the American College of Rheumatology revised criteria for the classification of systemic lupus erythematosus. Arthritis Rheum. 1997;40:1725.

15. Weening JJ, D'Agati VD, Schwartz MM, Seshan SV, Alpers CE, Appel GB, et al. The classification of glomerulonephritis in systemic lupus erythematosus revisited. Kidney Int. 2004;65:521-30.

16. Gladman DD, Ibañez D, Urowitz MB. Systemic lupus erythematosus disease activity index 2000. J Rheumatol. 2002;29:288-91.

17. Alwashih MA, Watson DG, Andrew R, Stimson RH, Alossaimi, M, Blackburn G, et al. Plasma metabolomic profile varies with glucocorticoid dose in patients with congenital adrenal hyperplasia. Sci Rep. 2017;7:17092.

18. Yuhara T, Takemura H, Akama T, Suzuki H, Yamane K, Kashiwagi, H. Predicting infection in hospitalized patients with systemic lupus erythematosus. Intern Med. 1996;35:629-36.

19. Fei Y, Shi X, Gan F, Li X, Zhang W, Li M, et al. Death causes and pathogens analysis of systemic lupus erythematosus during the past 26 years. Clin Rheumatol. 2014;33:57-63.

20. Feldman CH, Hiraki LT, Winkelmayer WC, Marty FM, Franklin JM, Kim SC, et al. Serious infections among adult Medicaid beneficiaries with systemic lupus erythematosus and lupus nephritis. Arthritis Rheumatol. 2015;67:1577-85.

21. Sisó A, Ramos-Casals M, Bové A, Brito-Zerón P, Soria N, Muñoz S, et al. Previous antimalarial therapy in patients diagnosed with lupus nephritis: influence on outcomes and survival. Lupus. 2008;17:281-8.

22. Chakravarty EF, Bush TM, Manzi S, Clarke AE, Ward MM. Prevalence of adult systemic lupus erythematosus in California and Pennsylvania in 2000: Estimates obtained using hospitalization data. Arthritis Rheum. 2007;56:2092-4. 
23. Fauci AS, Dale DC, Balow JE. Glucocorticosteroid therapy: mechanisms of action and clinical considerations. Ann Intern Med. 1976;84:304-15.

24. Herzer P, Lemmel EM. Inhibition of granulocyte function by prednisolone and non-steroid antiinflammatory drugs. Quantitative evaluation with nbt test and its correlation with phagocytosis. Immunobiology. 1980;157:78-88.

25. Feldman $\mathrm{CH}$, Marty FM, Winkelmayer WC, Guan H, Franklin JM, Solomon DH, et al. Comparative rates of serious infections among patients with systemic lupus erythematosus receiving immunosuppressive medications. Arthritis Rheumatol. 2017;69:387-97.

26. Stuck AE, Minder CE, Frey FJ. Risk of infectious complications in patients taking glucocorticosteroids. Rev Infect Dis. 1989;11:954-63.

27. Dixon WG, Abrahamowicz M, Beauchamp ME, Ray DW, Bernatsky S, Suissa S, et al. Immediate and delayed impact of oral glucocorticoid therapy on risk of serious infection in older patients with rheumatoid arthritis: a nested case-control analysis. Ann Rheum Dis. 2012;71:1128-33.

28. Luijten RK, Cuppen BVJ, Bijlsma JWJ, Derksen RH. Serious infections in systemic lupus erythematosus with a focus on pneumococcal infections. Lupus. 2014;23:1512-6.

29. Rezende RPV, Ribeiro FM, Albuquerque EMN, Gayer CR, Andrade LEC, Klumb EM. Immunogenicity of pneumococcal polysaccharide vaccine in adult systemic lupus erythematosus patients undergoing immunosuppressive treatment. Lupus. 2016;25:1254-9.

\section{Figures}

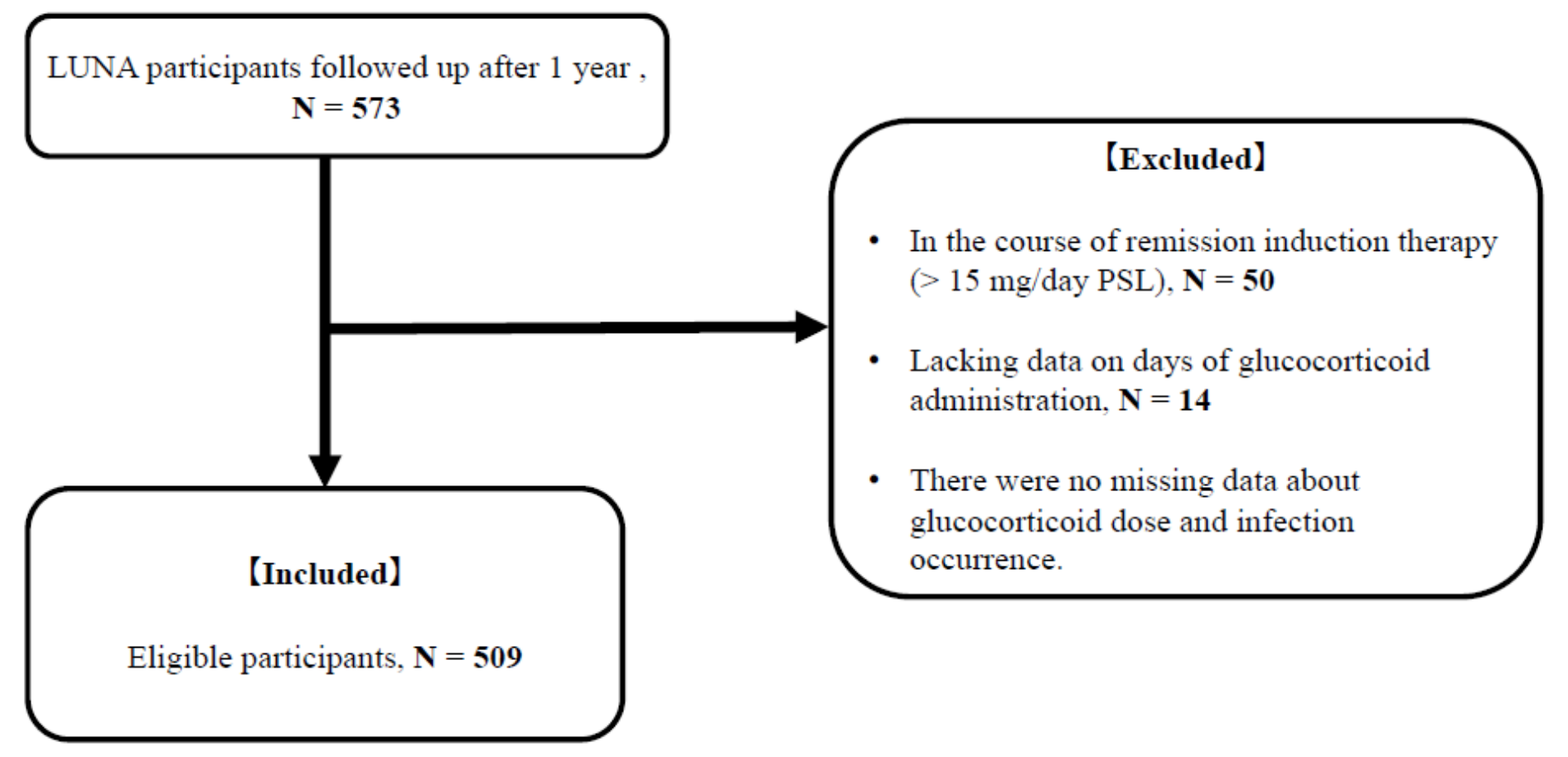

Figure 1 
Flowchart of eligible and ineligible participants A total of 573 LUNA participants were followed up after 1 year. The total number of excluded patients was 64 (50 patients who were undergoing remission therapy with PSL >15 mg; 14 patients with missing data on the number of days of glucocorticoid administration). There were no cases lacking data on glucocorticoid dose and infection occurrence. The final number of eligible participants was 509 .

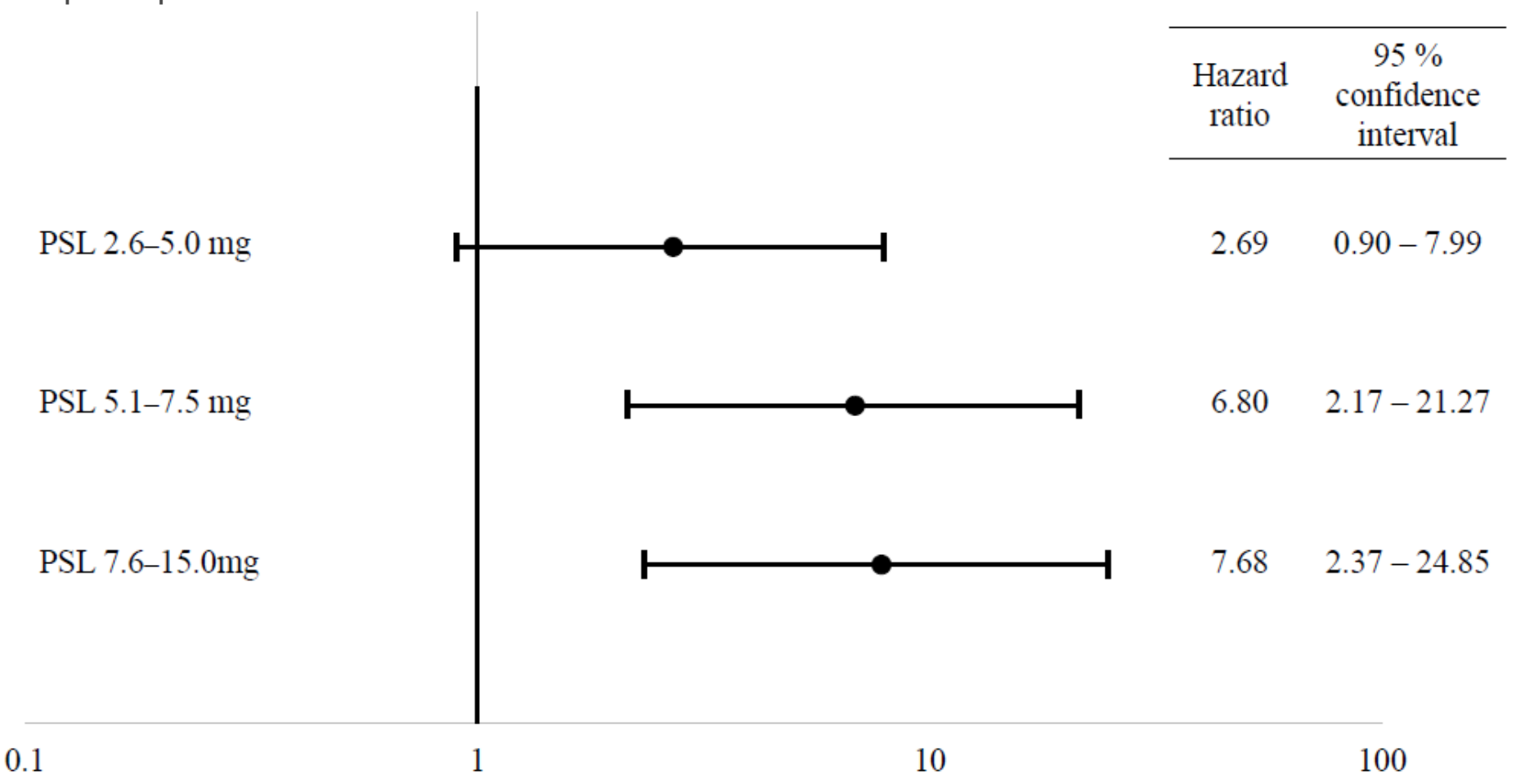

Adjusted hazard ratio for infection occurrence

Figure 2

Forest plot of the adjusted hazard ratio of infection occurrence 